\section{SP0150 FUNCTIONAL REHABILITATION FOR THE RELIEF OF COMPLEX PERSISTENT PAIN}

C. Mccabe. Royal National Hospital for Rheumatic Diseases, Royal United Hopsitals Nhs Foundation Trust, Bath, UK

Persistent pain, by definition, is a long-term condition that cannot be resolved by available medical or other treatments. Historically, the focus for pain and rehabilitation specialities has been on management, to improve quality of life and function in the presence of pain. However, most patients seek pain relief.

Research by the Bath Pain group, and other published literature indicates that people make hypotheses about bodily feelings based on prior performance and the current environment. This is an active process. Pain related adaptations to the sensory and motor systems ensure we act to minimise potential threats. These adaptations impact on bodily feelings and modify autonomic and limbic systems. The degree of adaptation can be minor and brief, or significant and persistent. Where adaptive mechanisms continue to engage beyond what is deemed as useful, this can be considered hyper-adaptive.

I will present clinical evidence of pain related hyper-adaptations that people with persistent pain describe in clinic. For example, perceived changes in the painful limb, ownership, temperature, and size, as well as feelings of peculiarity, discomfort, pain, and altered movement trajectory when motor output does not match expected sensory feedback. People with Rheumatoid Arthritis, Fibromyalgia, Complex Regional Pain Syndrome, or after limb fracture all describe altered body perceptions. Individuals may, for example, perceive a painful hand (and an object held within it), as excessively heavy and enlarged (when in reality it is not), report clumsiness, and difficulty moving the hand due to a perceived inability to 'engage' with it. We have described visual neglect and emotional changes about the painful limb, and consequent impacts on motor and autonomic function.

The sensory system encodes the characteristics and location of stimuli and determines if these are harmful or innocuous. This process is impaired in a painful body part. The ability to determine the texture or temperature of materials applied to the painful skin is commonly lost; or non-painful stimuli, such as light touch, are perceived as painful (allodynia).

This session will describe work that has focused on aiming to increase our understanding of the pain-related adaptations in sensorimotor processing and associated behaviours, in order to design interventions that help to redefine this hyperadaptive response, essentially broadening sensorimotor function, and relieving persistent pain.

Disclosure of Interest: None declared

DOI: 10.1136/annrheumdis-2018-eular.7877

SATURDAY, 16 JUNE 2018

Osteoarthritis: a vascular disease.

\section{SP0151 DOES IMAGING SUPPORT THE VASCULAR NATURE OF OSTEOARTHRITIS?}

P.G. Conaghan. Leeds Institute of Rheumatic and Musculoskeletal Medicine, University of Leeds, Leeds, UK

There are a number of ways in which vascular disease may contribute to either the initiation or structural progression of osteoarthritis (OA). We should keep in mind that atherosclerotic vascular disease, like OA, occurs with increasing frequency as people age and consequently understanding their inter-relationship and any potential causal relationship is dificult. Of interest is the suggested epidemiological links between cardiovascular mortality and OA, though again this is difficult to discern the casual part of the relationship. Links between vascular disease and osteoarthritis may of course be mediated through a common disease association, obesity. One hypothesis is that osteoarthritis is a differentiation disorder involving altered lipid metabolism.

It should also be remembered that articular cartilage is avascular, and it receives much of its nutrition from the subchondral bone or synovial fluid. Vascular disease in the subchondral bone may accelerate structual progression through alterations in cartilage nutrition or through direct ischaemic effects on bone. Imaging plays a key role in hypotheses about vascular involvement in OA, since it is commonly used for diagnosis and assessing progression. It has been suggested that the MRI subchondral bone changes typical of OA (termed bone marrow lesions) are similar to those seen in avascular necrosis, especially in the hip.

How might vascular changes effect OA? Certainly OA structural changes have been proposed because of secondary vascular changes in the high-pressure subchondral bone environment. These have been described in terms such as 'venous outflow obstruction' and also 'localised hypercoagulabilities', and have been described in animal and human studies. Venous outlet syndrome in the subchondral region can result in ischaemia of bone and the adjacent cartilage. Dynamic contrast enhanced (DCE-)MRI and positron emission tomography (PET) have demonstrated that venous outflow obstruction results in decreased perfusion and that venus staus is associated with trabecular remodelling, in animal OA models. Another potential role for vascular perturbation is in the synovitis commonly seen in OA. Given the frequency of synovitis in (especially knee) OA, it is possible that a patchy distribution of reduced blood flow (local ischaemia) happens in areas of greater inflammation. Greater levels of synovial inflammation have been associated with OA progression

Another hypothesis is that atheromatous disease might directly effect OA pathology progression. Computered tomography (CT) has been used in the spine to demonstrate an association between arterial calcification and the degree of disc degeneration, and there has also been shown to be a relationship between disc degeneration and adjacent bony end plate damage. These spinal changes are similar to $\mathrm{OA}$ joint pathologies. Another study demonstrated an association between hand osteoarthritis imaging findings and aortic calcification.

\section{REFERENCES :}

[1] Aaron RK, Racine J, Dyke J. Contribution of circulatroy disturbances in subchondral bone to the pathophysiology of osteoarthritis. Curr Rheumatol Rep 2017;19:49.

[2] Conaghan PG, Vanharanta $\mathrm{H}$, Dieppe PA. Is progressive osteoarthritis an atheromatous vascular disesase? Ann Rheum Dis 2005;64:1539-41.

Disclosure of Interest: None declared DOI: 10.1136/annrheumdis-2018-eular.7854

\section{SP0152 HYPERTENSION, BONE MARROW LESIONS AND OSTEOARTHRITIS (OA)}

${ }^{1,2}$ D. Felson. ${ }^{1}$ Clinical Epidemiology Unit, Boston University School of Medicine, Boston, USA; ${ }^{2}$ Arthritis Reseach UK Centre for Epidemiology, University of Manchester, Manchester, UK

Elements of the metabolic syndrome except for hypertension such as dyslipidemia and central adiposity are not consistently associated with knee osteoarthritis once obesity is adjusted for. However, after adjustment for obesity, hypertension is modestly associated with an increased risk of knee and even, in some studies, hand OA. A recent meta-analysis reported consistency across large cohort and case control studies; those with hypertension had a 1.49 fold increased odds of OA $(p<0.001)$

There a several possible explanations for this association especially if hypertension compromises the supply of nutrients to the joint. First, as OA develops, sub chondral bone remodelling is accompanied by angiogenesis which could be inhibited by vascular insufficiency. In findings that are probably related, bone marrow lesions seen on MRI in OA represent lesions of bone trauma and, in these lesions, the number of vessels increases and the vessels have especially thick walls for their size. Bone marrow lesions have been linked to intraosseous hypertension that is probably not a consequence of arterial but rather of venous hypertension within bone. Further, NSAID use in persons with OA could raise blood pressure and create a spurious association of $O A$ with hypertension. Given this range of potential explanations and the potential for identification of causal factors that may offer clues to OA treatment, further exploration of the biology of this relationship is needed.

Disclosure of Interest: None declared

DOI: 10.1136/annrheumdis-2018-eular.7863

\section{SATURDAY, 16 JUNE 2018}

\section{Workshop: Is there a diet for people with RMDs?}

\section{SP0153 WHAT EVIDENCE IS THERE THAT DIETS HELP PEOPLE} WITH RMDS?

${ }^{1,2}$ A. Linauskas. ${ }^{1}$ Department of Rheumatology, Aarhus University Hospital, Aarhus; ${ }^{2}$ Department of Rheumatology, North Denmark Regional Hospital, Hjoerring, Denmark

Throughout history patients with Rheumatic diseases (RMD) have used different diets trying to improve the symptoms and dietary manipulation is still widely used today. There are several potential mechanisms by which diet may be related to pathways involved in inflammation, such as decreasing the inflammatory process increasing antioxidant levels, changing the lipid profile, influencing composition of the intestinal bacterial flora.

The common dietary programs used by people with RMD include vegetarian or vegan, Mediterranean, elimination diets or fasting periods. Moreover, many 